\title{
Heterosis and Combining Ability Studies in Indigenous Collection of Pearl Millet Germplasm [Pennisetum glaucum (L.) R. Br.]
}

\author{
M. Bala Barathi ${ }^{*}$, B. Vijaya Lakshmi ${ }^{1}$, P. Sanjana Reddy ${ }^{2}$ and Sk. Nafeez Umar ${ }^{3}$
}

${ }^{1}$ Department of Genetics and Plant Breeding, Agricultural College, Bapatla-522101 Andhra Pradesh, India

${ }^{2}$ Indian Institute of Millets Research, Rajendranagar-500030, Hyderabad, Telangana, India

${ }^{3}$ Department of Statistics and Computer Applications, Agricultural College, Tirupathi517501, Andhra Pradesh, India

*Corresponding author

\section{A B S T R A C T}

\begin{tabular}{|l|}
\hline K e y w o r d s \\
$\begin{array}{l}\text { Heterosis, Grain } \\
\text { yield, Pearl millet } \\
\text { and line } \times \text { tester }\end{array}$ \\
\hline Article Info \\
\hline $\begin{array}{l}\text { Accepted: } \\
\text { 20 September } 2020 \\
\text { Available Online: } \\
\text { 10 October } 2020\end{array}$ \\
\hline
\end{tabular}

\section{Introduction}

Pearl millet [Pennisetum glaucum (L.) R. Br.] is the important staple food crop in arid and semi-arid zones. It occupied sixth place among cereal crops in the world after wheat, rice, maize, barley and sorghum. In India it occupies fourth place in acreage of 7.4 million hectares with a production of 9.2 million tonnes and productivity of $1231 \mathrm{~kg} / \mathrm{ha}$ (Ministry of Agriculture, 2017-2018). Pearl millet is a cross pollinated crop with protogynous nature and also availability of
The heterosis and combining ability study was conducted in pearl millet involving 60 crosses and 32 parents for 10 quantitative traits to predict the gene action involved in inheritance of yield and yield contributing traits and to identify best general combiners and superior crosses. Among parents, the line ICMA 04999 and testers 2325, 2396, 2306, 2337, 2348 and 2394 were the good general combiners for grain yield and could be used in hybridization programme to exploit their general combining ability. The components of variance due to gca and sca revealed predominance of non-additive gene action for all the traits. The cross ICMA $04999 \times 2309$ recorded high significant positive $s c a$ effect, mid parent, better parent heterotic effect and per se performance for grain yield. 
(Khandagale et al., 2014). So, combining ability analysis is necessary for selection of parents before conducting any hybrid breeding programme. Among the biometrical procedures, Line $\times$ Tester mating design is widely used to study the combining ability of parents chosen for heterosis breeding (Solanki et al., 2017). This design is helpful in evaluation of large number of germplasm lines at a time in terms of combining ability variances and effects (Sprague and Tatum, 1942).

Hence the present investigation was undertaken to study combining ability and heterosis for selection of desired hybrids based on their mid parent heterosis, heterobeltiosis and standard heterosis.

\section{Materials and Methods}

The material consists of two male sterile lines (ICMA 04999 and ICMA 97111), 30 testers collected from different parts of the India $i$.e., Tamilnadu, Maharashtra, Madhya Pradesh and Andhra Paradesh, six national checks (GHB 558, GHB 905, RHB 173, HHB 272, MPMH 21, HHB 67 improved) and $60 \mathrm{~F}_{1}$ hybrids generated by crossing two male sterile lines with 30 testers in Line $\times$ Tester mating design (Kempthorne, 1957) at IIMR during summer, 2019. All the $F_{1}$ 's along the parents and checks were raised in alpha lattice design (Patterson and Williams, 1976) with three replications during kharif, 2019. Each genotype was grown in two rows with two metres row length and spacing of $45 \mathrm{~cm}$ between rows and $15 \mathrm{~cm}$ between plants in a row. All agronomic and plant protection measures were followed as per recommendation to raise good and healthy crop. The observations were recorded on five randomly selected competitive plants in each replication for 10 quantitative traits viz., days to 50 per cent flowering, plant height, total number of productive tillers, panicle length, panicle width, 1000-grain weight, fodder yield, grain yield, total biomass and harvest index. Among the six checks, the per se performance of RHB- 173 was good and this was taken as standard parent for estimation of standard heterosis. Statistical analysis was executed using Genstat 12 edn and Indostat software packages.

\section{Results and Discussion}

The Analysis of variance for combining ability analysis revealed the presence of considerable amount of genetic variability in the experimental material for all the traits except total number of productive tillers per plant indicating significant contribution of these traits towards combining ability. The components of variance for all the characters suggested that the gca variance was less than the $s c a$ variance, the ratio of $g c a / s c a$ variance being less than unity indicating the predominance of non- additive gene action in the inheritance of these traits. This indicates that recurrent selection for specific combining ability would be quite effective in improvement of these traits. Similar pattern of results for non-additive gene action were earlier reported by Bharath and Dangaria (2018) for days to 50 per cent flowering, Gavali et al., (2018) for plant height, panicle length and panicle width; Patel et al., (2018) for 1000- grain weight, Krishnan et al., (2019) for number of productive tillers per plant and grain yield; Shinde and Mehetre (2014) for fodder yield; Solanki et al., (2017) for total biomass and harvest index. (Table 1).

The general combining ability estimates provide the information regarding the average performance of a line among different crosses which in turn reflects the breeding value of the line. In the present study, general combining ability effects of parents revealed that none of the line or tester recorded significant gca effect in desirable direction for 
all the traits. However, among the parents, the tester 2325 was turned to be the good general combiner for grain yield, panicle width in addition to panicle length and 1000-grain weight in desirable direction. The parent 2381 and 2349 recorded highest significant gca effect in desirable direction for days to 50 per cent flowering and plant height respectively, which inturn can be used in breeding programmes to develop early and dwarf genotypes. The tester 2368 was good combiner for panicle length along with 1000grain weight. Similarly the parents 2352 and 2365 were found to be good combiners for 1000-grain weight and harvest index respectively. Athoni et al., (2016) reported significant gca effects for days to 50 per cent flowering, plant height, panicle length, panicle width, 1000 grain weight and grain yield; Kumar et al., (2017) for harvest index (Table 2).

Consideration of per se performance of parents along with $\mathrm{gca}$ effects will give better results in selection of parents for hybrid breeding programme (Rao, 1972 and Bhadalia et al., 2014). In the present study the parents 2348, 2325, 2306 and 2394 recorded significant $g c a$ effects for grain yield in desirable direction along with better per se performance. The results are in accordance with earlier reports of Bhardwaj et al., (2015) for grain yield. Therefore, the crosses involving 2325, 2381 and 2349 would result in development of good hybrids with favourable gene combinations for grain yield, days to 50 per cent flowering and plant height respectively.

The specific combining ability effects determine the specific cross combination for a particular trait or group of traits. A perusal of sca effects (Table 3) revealed that the cross ICMA $97111 \times 2386$ was best specific combiner for days to 50 per cent flowering. This cross may be further used in breeding programs for improvement of this trait and for the production of short duration hybrids. ICMA $04999 \times 2382$ and ICMA $97111 \times$ 2311 recorded high significant and positive sca effects for the traits viz., plant height and panicle length. The crosses ICMA $04999 \times$ 2311 and ICMA $97111 \times 2382$ recorded significant negative $s c a$ effects as these were best for improvement of short genotypes with lodging resistance. The cross ICMA $04999 \times$ 2309 was best specific combiner for grain yield and fodder yield. The crosses ICMA $04999 \times 2348$ and ICMA $97111 \times 2348$ recorded highest significant sca effect with a common tester for total biomass and harvest index respectively. The crosses ICMA 04999 $\times 2310$ and ICMA $97111 \times 2310$ were best for days to 50 per cent flowering and fodder yield with a common tester respectively. It indicates that, even though the two crosses have same tester, the two traits were not best in one cross. Similar results for significant sca effects in desirable direction were earlier reported by Singh and Sharma (2014) for plant height, panicle length and panicle width; Saini et al., (2018) for days to 50 per cent flowering, total biomass, harvest index and grain yield.

The crosses with high sca effects resulting from low $\times$ low gca parental combinations was observed in ICMA $97111 \times 2386$ for days to 50 per cent flowering, ICMA $97111 \times$ 2311 for plant height, ICMA $04999 \times 2382$ for panicle length, ICMA $97111 \times 2310$ for fodder yield, ICMA $04999 \times 2309$ for grain yield indicating the involvement of complimentary gene action in the inheritance of these traits. The crosses from high $\times$ low or low $\times$ high $g c a$ parental combinations with high sca effects were noticed in ICMA 97111 $\times 2333$ for 1000-grain weight; ICMA $04999 \times$ 2348 for total biomass and ICMA $97111 \times$ 2348 for harvest index indicating that the involvement of one low combiner will result in high sca effects. 
Table.1 Analysis of variances for combining ability for yield and its component characters in Pearl millet [Pennisetum glaucum (L.) R. Br.]

\begin{tabular}{|c|c|c|c|c|c|c|c|c|c|c|c|}
\hline $\begin{array}{l}\text { Source of } \\
\text { variations }\end{array}$ & d.f. & $\begin{array}{l}\text { Days to } 50 \\
\text { per cent } \\
\text { flowering }\end{array}$ & $\begin{array}{l}\text { Plant height } \\
\text { (cm) }\end{array}$ & $\begin{array}{l}\text { Total number } \\
\text { of productive } \\
\text { tillers per } \\
\text { plant }\end{array}$ & $\begin{array}{l}\text { Panicle } \\
\text { length }(\mathrm{cm})\end{array}$ & $\begin{array}{l}\text { Panicle } \\
\text { width }(\mathrm{cm})\end{array}$ & $\begin{array}{l}1000 \text { grain } \\
\text { weight }(g)\end{array}$ & $\begin{array}{l}\text { Fodder } \\
\text { yield (t/ha) }\end{array}$ & $\begin{array}{l}\text { Total } \\
\text { biomass } \\
\text { (t/ha) }\end{array}$ & $\begin{array}{l}\text { Harvest } \\
\text { index }\end{array}$ & $\begin{array}{l}\text { Grain } \\
\text { yield } \\
(t / h a)\end{array}$ \\
\hline Replications & 2 & $54.551 *$ & $2158.097 * *$ & 0.630 & 5.946 & 0.055 & 0.999 & $156.327^{*}$ & $105.576 * *$ & $0.061 * *$ & 2.011 \\
\hline Treatments & 59 & $33.465^{* *}$ & $665.422 * *$ & 0.593 & $18.232 * *$ & $0.197 * *$ & $4.913 * *$ & $86.406 * *$ & $37.280 * *$ & $0.008 * *$ & $1.541 * *$ \\
\hline Line effect & 1 & 14.735 & 40.850 & 0.118 & 13.484 & 0.017 & 1.842 & 35.506 & 126.085 & 0.012 & 1.140 \\
\hline Tester effect & 29 & 18.524 & 487.630 & 0.714 & 22.648 & 0.243 & 5.651 & 83.875 & 37.396 & 0.007 & 1.854 \\
\hline $\begin{array}{l}\text { Lines } \times \text { Tester } \\
\text { effect }\end{array}$ & 29 & $49.051 * *$ & $864.751 * *$ & 0.489 & $13.979 * *$ & $0.158 * *$ & $4.281 * *$ & $90.693 * *$ & $34.103 * *$ & 0.008 & $1.242 *$ \\
\hline Error & 118 & 7.560 & 298.979 & 0.659 & 3.785 & 0.070 & 1.026 & 37.085 & 15.042 & 0.004 & 0.761 \\
\hline$\sigma^{2}$ gca & & 0.1890 & -0.7237 & -0.0051 & $0.2975^{*}$ & 0.0012 & 0.0567 & 0.471 & $1.3895^{*}$ & 0.0001 & 0.0153 \\
\hline$\sigma^{2}$ sca & & $13.8303 * *$ & $188.5907 * *$ & -0.0569 & $3.3982 * *$ & $0.0293 * *$ & $1.0850 * *$ & $17.8694 * *$ & $6.3534 * *$ & $0.0014 * *$ & $0.1602 *$ \\
\hline$\sigma^{2}$ gca / $\sigma^{2}$ sca & & 0.0137 & -0.004 & 0.0896 & 0.0875 & 0.0409 & 0.0522 & 0.0263 & 0.218 & 0.0714 & 0.0955 \\
\hline
\end{tabular}

* Significant at $5 \%$ level; ** Significant at $1 \%$ level

Table.2 General combining ability for quantitative traits in pearl millet

\begin{tabular}{|c|c|c|c|c|c|c|c|c|c|c|}
\hline Parents & $\begin{array}{l}\text { Days to } 50 \\
\text { per cent } \\
\text { flowering }\end{array}$ & $\begin{array}{l}\text { Plant height } \\
\text { (cm) }\end{array}$ & $\begin{array}{l}\text { Total number } \\
\text { of productive } \\
\text { tillers per } \\
\text { plant }\end{array}$ & $\begin{array}{l}\text { Panicle } \\
\text { length }(\mathrm{cm})\end{array}$ & $\begin{array}{l}\text { Panicle } \\
\text { width }(\mathrm{cm})\end{array}$ & $\begin{array}{l}1000 \text { grain } \\
\text { weight (g) }\end{array}$ & $\begin{array}{l}\text { Fodder } \\
\text { yield (t/ha) }\end{array}$ & $\begin{array}{l}\text { Total } \\
\text { biomass } \\
\text { (t/ha) }\end{array}$ & $\begin{array}{l}\text { Harvest } \\
\text { index }\end{array}$ & $\begin{array}{l}\text { Grain yield } \\
\text { (t/ha) }\end{array}$ \\
\hline \multicolumn{11}{|l|}{ Lines } \\
\hline $\begin{array}{l}\text { ICMA } \\
04999\end{array}$ & $-0.286 * *$ & 0.476 & $-0.026 * *$ & $0.274^{*}$ & $0.010 * *$ & $-0.101 * *$ & 0.444 & $0.837^{*}$ & $-0.008 * *$ & $0.080 * *$ \\
\hline $\begin{array}{l}\text { ICMA } \\
97111\end{array}$ & 0.286 & $-0.476 * *$ & 0.026 & $-0.274 * *$ & $-0.010 * *$ & $0.101 * *$ & $-0.444 * *$ & $-0.837 * *$ & $0.008 * *$ & $-0.080 * *$ \\
\hline
\end{tabular}




\begin{tabular}{|c|c|c|c|c|c|c|c|c|c|c|}
\hline SE & 0.289 & 1.822 & 0.085 & 0.205 & 0.027 & 0.106 & 0.641 & 0.4088 & 0.0071 & 0.092 \\
\hline C D at $5 \%$ & 0.573 & 3.609 & 0.169 & 0.406 & 0.055 & 0.211 & 1.271 & 0.8096 & 0.0140 & 0.182 \\
\hline \multicolumn{11}{|l|}{ Testers } \\
\hline 2306 & 0.914 & 7.015 & 0.356 & 1.543 & $0.214 * *$ & $1.701 * *$ & 6.475 & 3.530 & $-0.008 * *$ & $0.724^{*}$ \\
\hline 2309 & $-2.419 * *$ & $-12.418 * *$ & $-0.628 * *$ & $-2.207 * *$ & $-0.136 * *$ & $1.551 * *$ & 2.592 & $-0.550 * *$ & $-0.018^{* *}$ & $-0.159 * *$ \\
\hline 2310 & $-1.253 * *$ & $-7.118 * *$ & $-0.078 * *$ & $-1.441 * *$ & $-0.403 * *$ & $-1.732 * *$ & 2.409 & 1.090 & $-0.051 * *$ & $-0.276^{* *}$ \\
\hline 2311 & $-0.753 * *$ & 13.382 & $0.689 *$ & $-4.091 * *$ & $-0.553 * *$ & $-1.066^{* *}$ & $-2.791 * *$ & $-3.463 * *$ & $-0.036^{* *}$ & $-0.909 * *$ \\
\hline 2368 & 0.914 & 11.382 & 0.372 & $3.843 *$ & $0.064 *$ & $0.751 *$ & 6.292 & 4.117 & $-0.043 * *$ & 0.324 \\
\hline 2370 & 0.331 & $-8.318 * *$ & $-0.028 * *$ & $-3.707 * *$ & $-0.203 * *$ & $1.00 *$ & $-8.441 * *$ & $-4.526 * *$ & $-0.016 * *$ & $-1.176^{* *}$ \\
\hline 2318 & $-2.253 * *$ & $-12.451 * *$ & 0.372 & $-1.507 * *$ & $-0.303 * *$ & $-0.216 * *$ & $-4.441 * *$ & $-4.705 * *$ & $-0.001 * *$ & $-1.193 * *$ \\
\hline 2381 & $-5.086 * *$ & 7.599 & 0.356 & $-0.474 * *$ & $-0.036 * *$ & 0.568 & $-4.075^{* *}$ & $-1.541 * *$ & $-0.001 * *$ & $-0.176^{* *}$ \\
\hline 2325 & 0.247 & 10.065 & $-0.028 * *$ & $2.543^{*}$ & $0.298 * *$ & $0.684^{*}$ & 3.875 & 5.709 & $-0.019 * *$ & $0.874 * *$ \\
\hline 2327 & 1.081 & 3.382 & $-0.094 * *$ & $-2.141 * *$ & 0.014 & $-0.616^{* *}$ & $-1.125^{* *}$ & $-2.058 * *$ & $0.034 * *$ & $-0.059 * *$ \\
\hline 2328 & $-0.086 * *$ & $-3.418 * *$ & $-0.361 * *$ & $-0.291 * *$ & $-0.003 * *$ & $-0.016 * *$ & 3.909 & 1.634 & 0.001 & 0.207 \\
\hline 2329 & $-0.419 * *$ & 7.565 & $-0.294 * *$ & $-0.424 * *$ & $0.064 *$ & $-0.916 * *$ & 1.475 & 0.904 & $0.007 * *$ & 0.441 \\
\hline 2330 & 1.081 & 18.349 & 0.256 & 1.343 & $0.198 * *$ & $-0.266 * *$ & 0.175 & 1.722 & $0.009 * *$ & 0.207 \\
\hline 2331 & 1.581 & 2.549 & $-0.094 * *$ & 1.976 & $-0.103 * *$ & $-0.466 * *$ & $-4.808 * *$ & $-3.801 * *$ & $0.031 * *$ & $-0.409 * *$ \\
\hline 2332 & 3.414 & 0.249 & $-0.044 * *$ & 1.926 & $0.098 * *$ & $-1.182 * *$ & $-0.375^{* *}$ & $-1.093 * *$ & $0.012 * *$ & $-0.209 * *$ \\
\hline 2333 & $-0.25^{* *}$ & 1.732 & $-0.011 * *$ & $-0.524 * *$ & $0.131 * *$ & $1.551 * *$ & $-1.475 * *$ & $-0.803 * *$ & $0.036 * *$ & 0.391 \\
\hline 2337 & $-2.753 * *$ & $-6.618 * *$ & 0.089 & $-1.957 * *$ & $0.131 * *$ & $-0.549 * *$ & 1.859 & 2.187 & $0.006 * *$ & $0.724^{*}$ \\
\hline 2382 & 2.414 & 14.732 & $-0.078 * *$ & 0.809 & $0.114 * *$ & 0.001 & 3.692 & 2.422 & $-0.058 * *$ & $-0.476 * *$ \\
\hline 2342 & $-0.919 * *$ & 0.015 & 0.206 & $-1.941 * *$ & $-0.269 * *$ & $-1.132 * *$ & 4.092 & 0.000 & $-0.024 * *$ & $-0.176 * *$ \\
\hline 2386 & 1.247 & 4.415 & $0.472 *$ & $-0.624 * *$ & $0.131 * *$ & $-1.216 * *$ & 3.342 & 2.002 & $-0.044 * *$ & $-0.176^{* *}$ \\
\hline 2348 & 1.747 & 1.757 & 0.256 & 1.781 & $-0.061 * *$ & $-0.631 * *$ & 0.651 & 1.447 & $0.067 * *$ & $0.686 *$ \\
\hline 2352 & $-1.086 * *$ & $-4.151 * *$ & $-0.261 * *$ & $-0.991 * *$ & $0.148 * *$ & $1.901 * *$ & $-0.558 * *$ & $-1.190 * *$ & $0.012 * *$ & $-0.009 * *$ \\
\hline 2364 & 0.914 & $-8.285^{* *}$ & 0.139 & $3.643 *$ & $0.164 * *$ & 0.218 & 5.375 & 1.569 & $-0.001 * *$ & 0.474 \\
\hline 2365 & 0.914 & $-6.601 * *$ & $-0.361 * *$ & $-0.191 * *$ & $0.081 * *$ & $-0.049 * *$ & $-3.141 * *$ & $-2.880 * *$ & $0.086 * *$ & 0.041 \\
\hline 2387 & $-0.086 * *$ & $-12.385 * *$ & $-0.311 * *$ & 0.459 & $-0.269 * *$ & $-1.466 * *$ & $-0.341 * *$ & $-0.205^{* *}$ & $-0.023 * *$ & $-0.176 * *$ \\
\hline 2394 & $-2.419 * *$ & $-4.151 * *$ & $-0.128 * *$ & 1.359 & $0.098 * *$ & 0.601 & $-4.258 * *$ & 0.152 & $0.046 * *$ & $0.674^{*}$ \\
\hline 2395 & 0.414 & $-1.651 * *$ & $-0.028 * *$ & 0.109 & $-0.053 * *$ & $-0.166^{* *}$ & $-1.308 * *$ & 0.224 & $-0.021 * *$ & $-0.159 * *$ \\
\hline 2396 & 1.081 & 5.015 & $-0.811 * *$ & 1.693 & $0.148 * *$ & 0.401 & $-2.225^{* *}$ & 0.167 & $0.056 * *$ & $0.824 *$ \\
\hline 2346 & 1.747 & $-2.485 * *$ & $0.489^{*}$ & 0.076 & $0.098 * *$ & 0.201 & $-4.825^{* *}$ & $-2.056^{* *}$ & $-0.013 * *$ & $-0.643 * *$ \\
\hline 2349 & $-0.253 * *$ & $-19.151 * *$ & $-0.411 * *$ & $-0.591 * *$ & 0.198** & 0.551 & $-2.025^{* *}$ & $-0.003 * *$ & $-0.028 * *$ & $-0.209 * *$ \\
\hline SE & 1.122 & 7.059 & 0.331 & 0.794 & 0.108 & 0.413 & 2.486 & 1.583 & 0.027 & 0.356 \\
\hline CD at $5 \%$ & 2.222 & 13.978 & 0.656 & 1.572 & 0.214 & 0.818 & 4.923 & 3.135 & 0.054 & 0.705 \\
\hline
\end{tabular}

* Significant at $5 \%$ level; ** Significant at $1 \%$ level 
Table.3 Specific combining ability for quantitative traits in pearl millet

\begin{tabular}{|c|c|c|c|c|c|c|c|c|c|c|}
\hline Crosses & $\begin{array}{l}\text { Days to } 50 \\
\text { per cent } \\
\text { flowering }\end{array}$ & $\begin{array}{l}\text { Plant height } \\
\text { (cm) }\end{array}$ & $\begin{array}{l}\text { Total number } \\
\text { of productive } \\
\text { tillers per plant }\end{array}$ & $\begin{array}{l}\text { Panicle } \\
\text { length }(\mathrm{cm})\end{array}$ & $\begin{array}{l}\text { Panicle } \\
\text { width }(\mathrm{cm})\end{array}$ & $\begin{array}{l}1000 \text { grain } \\
\text { weight }(g)\end{array}$ & $\begin{array}{l}\text { Fodder yield } \\
\text { (t/ha) }\end{array}$ & $\begin{array}{l}\text { Total } \\
\text { biomass } \\
\text { (t/ha) }\end{array}$ & $\begin{array}{l}\text { Harvest } \\
\text { index }\end{array}$ & $\begin{array}{l}\text { Grain } \\
\text { yield } \\
\text { (t/ha) }\end{array}$ \\
\hline ICMA $04999 \times 2306$ & -0.381 & -1.643 & -0.524 & 1.310 & -0.176 & -0.316 & 0.106 & 0.236 & -0.005 & -0.063 \\
\hline ICMA $04999 \times 2309$ & 1.953 & 6.190 & 0.359 & 0.960 & 0.140 & $-1.199 *$ & $7.323 *$ & 3.506 & 0.038 & $1.120 *$ \\
\hline ICMA $04999 \times 2310$ & $-5.547 * *$ & -14.176 & -0.191 & -0.274 & -0.093 & 0.751 & $-8.761 *$ & -2.967 & 0.038 & 0.004 \\
\hline ICMA $04999 \times 2311$ & $-5.047 * *$ & $-34.610 * *$ & -0.524 & $-2.657 *$ & 0.090 & 0.218 & $-7.294 *$ & -3.924 & 0.010 & -0.730 \\
\hline ICMA $04999 \times 2368$ & $3.286^{*}$ & 3.990 & 0.259 & -0.457 & -0.260 & -0.399 & -3.044 & -0.574 & -0.040 & -0.730 \\
\hline ICMA $04999 \times 2370$ & 0.536 & 18.690 & 0.192 & 0.326 & 0.240 & -0.282 & 5.423 & 3.600 & 0.010 & 0.604 \\
\hline ICMA $04999 \times 2318$ & $-3.214^{*}$ & -3.776 & 0.359 & -0.540 & 0.140 & -0.999 & -1.211 & 1.168 & 0.012 & 0.187 \\
\hline ICMA $04999 \times 2381$ & 0.953 & -12.060 & 0.242 & -1.640 & -0.060 & -0.416 & -3.044 & -0.479 & 0.012 & 0.004 \\
\hline ICMA $04999 \times 2325$ & 1.619 & -1.360 & 0.426 & -1.924 & -0.193 & -0.932 & 1.973 & 2.801 & -0.023 & -0.080 \\
\hline ICMA $04999 \times 2327$ & 1.119 & -11.276 & 0.026 & -0.774 & -0.143 & 0.201 & 1.039 & -1.809 & -0.033 & -0.646 \\
\hline ICMA $04999 \times 2328$ & 1.619 & -1.410 & -0.241 & -0.190 & 0.074 & 0.501 & -0.627 & -3.084 & 0.073 & 0.287 \\
\hline ICMA $04999 \times 2329$ & 0.286 & 14.574 & -0.241 & -0.057 & 0.040 & -0.932 & -1.561 & -2.827 & 0.017 & -0.480 \\
\hline ICMA $04999 \times 2330$ & 0.453 & -1.310 & 0.176 & -1.890 & -0.193 & 0.551 & -5.427 & -4.302 & 0.052 & -0.380 \\
\hline ICMA $04999 \times 2331$ & $-4.714 * *$ & -7.176 & 0.026 & 0.743 & 0.007 & $1.385^{*}$ & -0.444 & -0.359 & 0.007 & -0.096 \\
\hline ICMA $04999 \times 2332$ & 0.453 & 0.124 & 0.076 & -1.074 & -0.126 & 0.301 & 4.356 & 0.306 & -0.055 & -0.296 \\
\hline ICMA $04999 \times 2333$ & 1.119 & 8.640 & -0.024 & 1.176 & -0.026 & $-2.232 * *$ & -4.144 & -1.354 & 0.052 & 0.270 \\
\hline ICMA $04999 \times 2337$ & -0.047 & 0.324 & -0.358 & 0.010 & 0.140 & 0.568 & -2.677 & -1.044 & -0.028 & -0.630 \\
\hline ICMA $04999 \times 2382$ & $4.453 * *$ & $27.307 * *$ & 0.142 & $3.710 * *$ & 0.090 & -0.149 & 3.989 & 3.181 & -0.065 & -0.530 \\
\hline ICMA $04999 \times 2342$ & -0.547 & 0.357 & 0.059 & 2.126 & 0.274 & 0.851 & -0.077 & 0.343 & 0.015 & 0.337 \\
\hline ICMA $04999 \times 2386$ & $6.619 * *$ & 1.024 & -0.074 & 1.543 & 0.174 & 0.701 & 6.206 & 2.788 & -0.018 & 0.137 \\
\hline ICMA $04999 \times 2348$ & 0.786 & 11.949 & -0.524 & 1.771 & -0.001 & 0.550 & 5.197 & $4.716^{*}$ & $-0.087 *$ & 0.508 \\
\hline ICMA $04999 \times 2352$ & -2.381 & 1.190 & 0.192 & -1.057 & -0.143 & 0.118 & 3.473 & 1.370 & -0.018 & 0.270 \\
\hline ICMA $04999 \times 2364$ & -3.047 & -6.343 & -0.074 & 0.343 & -0.160 & 0.968 & -1.361 & 0.131 & -0.032 & -0.280 \\
\hline ICMA $04999 \times 2365$ & 1.286 & 13.640 & 0.292 & 1.610 & -0.176 & -0.232 & -1.744 & -0.160 & -0.032 & -0.013 \\
\hline ICMA $04999 \times 2387$ & -0.381 & -8.810 & 0.242 & -0.940 & 0.174 & 0.984 & 2.156 & 1.785 & 0.007 & 0.470 \\
\hline ICMA $04999 \times 2394$ & 2.286 & 2.857 & -0.041 & -1.607 & 0.274 & $-1.749 * *$ & 3.073 & 1.255 & 0.018 & 0.554 \\
\hline ICMA $04999 \times 2395$ & $-4.547 * *$ & -16.310 & 0.292 & $-2.357 *$ & -0.210 & 0.851 & -2.477 & -1.047 & 0.058 & 0.487 \\
\hline ICMA $04999 \times 2396$ & -2.214 & -9.643 & -0.024 & -0.607 & -0.110 & 0.085 & -1.561 & -1.027 & 0.032 & 0.170 \\
\hline
\end{tabular}




\begin{tabular}{|c|c|c|c|c|c|c|c|c|c|c|}
\hline Crosses & $\begin{array}{l}\text { Days to } 50 \\
\text { per cent } \\
\text { flowering }\end{array}$ & $\begin{array}{l}\text { Plant height } \\
\text { (cm) }\end{array}$ & $\begin{array}{l}\text { Total } \\
\text { number of } \\
\text { productive } \\
\text { tillers per } \\
\text { plant }\end{array}$ & $\begin{array}{l}\text { Panicle } \\
\text { length }(\mathrm{cm})\end{array}$ & $\begin{array}{l}\text { Panicle } \\
\text { width }(\mathrm{cm})\end{array}$ & $\begin{array}{l}1000 \text { grain } \\
\text { weight (g) }\end{array}$ & $\begin{array}{l}\text { Fodder yield } \\
\text { (t/ha) }\end{array}$ & $\begin{array}{l}\text { Total biomass } \\
\text { (t/ha) }\end{array}$ & Harvest index & $\begin{array}{l}\text { Grain yield } \\
(\mathrm{t} / \mathrm{ha})\end{array}$ \\
\hline ICMA $04999 \times 2346$ & $3.453^{*}$ & 9.524 & -0.558 & -0.124 & 0.240 & -0.149 & 1.773 & 1.286 & -0.030 & 0.004 \\
\hline ICMA $04999 \times 2349$ & -0.214 & 9.524 & 0.042 & $2.543^{*}$ & -0.026 & 0.401 & -0.627 & -3.520 & 0.018 & -0.463 \\
\hline ICMA $97111 \times 2306$ & 0.381 & 1.643 & 0.524 & -1.310 & 0.176 & 0.316 & -0.106 & -0.236 & 0.005 & 0.063 \\
\hline ICMA $97111 \times 2309$ & -1.953 & -6.190 & -0.359 & -0.960 & -0.140 & $1.199 *$ & $-7.323 *$ & -3.506 & -0.038 & $-1.120 *$ \\
\hline ICMA $97111 \times 2310$ & $5.547 * *$ & 14.176 & 0.191 & 0.274 & 0.093 & -0.751 & $8.761^{*}$ & 2.967 & -0.038 & -0.004 \\
\hline ICMA $97111 \times 2311$ & $5.047 * *$ & $34.610 * *$ & 0.524 & $2.657 *$ & -0.090 & -0.218 & 7.294* & 3.924 & -0.010 & 0.730 \\
\hline ICMA $97111 \times 2368$ & $-3.286^{*}$ & -3.990 & -0.259 & 0.457 & 0.260 & 0.399 & 3.044 & 0.574 & 0.040 & 0.730 \\
\hline ICMA $97111 \times 2370$ & -0.536 & -18.690 & -0.192 & -0.326 & -0.240 & 0.282 & -5.423 & -3.600 & -0.010 & -0.604 \\
\hline ICMA $97111 \times 2318$ & $3.214^{*}$ & 3.776 & -0.359 & 0.540 & -0.140 & 0.999 & 1.211 & -1.168 & -0.012 & -0.187 \\
\hline ICMA $97111 \times 2381$ & -0.953 & 12.060 & -0.242 & 1.640 & 0.060 & 0.416 & 3.044 & 0.479 & -0.012 & -0.004 \\
\hline ICMA $97111 \times 2325$ & -1.619 & 1.360 & -0.426 & 1.924 & 0.193 & 0.932 & -1.973 & -2.801 & 0.023 & 0.080 \\
\hline ICMA $97111 \times 2327$ & -1.119 & 11.276 & -0.026 & 0.774 & 0.143 & -0.201 & -1.039 & 1.809 & 0.033 & 0.646 \\
\hline ICMA $97111 \times 2328$ & -1.619 & 1.410 & 0.241 & 0.190 & -0.074 & -0.501 & 0.627 & 3.084 & -0.073 & -0.287 \\
\hline ICMA $97111 \times 2329$ & -0.286 & -14.574 & 0.241 & 0.057 & -0.040 & 0.932 & 1.561 & 2.827 & -0.017 & 0.480 \\
\hline ICMA $97111 \times 2330$ & -0.453 & 1.310 & -0.176 & 1.890 & 0.193 & -0.551 & 5.427 & 4.302 & -0.052 & 0.380 \\
\hline ICMA $97111 \times 2331$ & $-4.714 * *$ & 7.176 & -0.026 & -0.743 & -0.007 & $-1.385^{*}$ & 0.444 & 0.359 & -0.007 & 0.096 \\
\hline ICMA $97111 \times 2332$ & -0.453 & -0.124 & -0.076 & 1.074 & 0.126 & -0.301 & -4.356 & -0.306 & 0.055 & 0.296 \\
\hline ICMA $97111 \times 2333$ & -1.119 & -8.640 & 0.024 & -1.176 & 0.026 & $2.232 * *$ & 4.144 & 1.354 & -0.052 & -0.270 \\
\hline ICMA $97111 \times 2337$ & 0.047 & -0.324 & 0.358 & -0.010 & -0.140 & -0.568 & 2.677 & 1.044 & 0.028 & 0.630 \\
\hline ICMA $97111 \times 2382$ & $-4.453 * *$ & $-27.307 * *$ & -0.142 & $-3.710 * *$ & -0.090 & 0.149 & -3.989 & -3.181 & 0.065 & 0.530 \\
\hline ICMA $97111 \times 2342$ & 0.547 & -0.357 & -0.059 & -2.126 & -0.274 & -0.851 & 0.077 & -0.343 & -0.015 & -0.337 \\
\hline ICMA $97111 \times 2386$ & $-6.619 * *$ & -1.024 & 0.074 & -1.543 & -0.174 & -0.701 & -6.206 & -2.788 & 0.018 & -0.137 \\
\hline ICMA $97111 \times 2348$ & -0.786 & -11.949 & 0.524 & -1.771 & 0.001 & -0.550 & -5.197 & $-4.716^{*}$ & $0.087^{*}$ & -0.508 \\
\hline ICMA $97111 \times 2352$ & 2.381 & -1.190 & -0.192 & 1.057 & 0.143 & -0.118 & -3.473 & -1.370 & 0.018 & -0.270 \\
\hline ICMA $97111 \times 2364$ & 3.047 & 6.343 & 0.074 & -0.343 & 0.160 & -0.968 & 1.361 & -0.131 & 0.032 & 0.280 \\
\hline ICMA $97111 \times 2365$ & -1.286 & -13.640 & -0.292 & -1.610 & 0.176 & 0.232 & 1.744 & 0.160 & 0.032 & 0.013 \\
\hline ICMA $97111 \times 2387$ & 0.381 & 8.810 & -0.242 & 0.940 & -0.174 & -0.984 & -2.156 & -1.785 & -0.007 & -0.470 \\
\hline ICMA $97111 \times 2394$ & -2.286 & -2.857 & 0.041 & 1.607 & -0.274 & $1.749 * *$ & -3.073 & -1.255 & -0.018 & -0.554 \\
\hline ICMA $97111 \times 2395$ & $4.547 * *$ & 16.310 & -0.292 & $2.357^{*}$ & 0.210 & -0.851 & 2.477 & 1.047 & -0.058 & -0.487 \\
\hline ICMA $97111 \times 2396$ & 2.214 & 9.643 & 0.024 & 0.607 & 0.110 & -0.085 & 1.561 & 1.027 & -0.032 & -0.170 \\
\hline ICMA $97111 \times 2346$ & $-3.453 *$ & -9.524 & 0.558 & 0.124 & -0.240 & 0.149 & -1.773 & -1.286 & 0.030 & -0.004 \\
\hline ICMA $97111 \times 2349$ & -0.214 & -9.524 & -0.042 & $-2.543 *$ & 0.026 & -0.401 & 0.627 & 3.520 & -0.018 & 0.463 \\
\hline SE & 1.587 & 9.983 & 0.468 & 1.123 & 0.153 & 0.584 & 3.515 & 2.239 & 0.038 & 0.503 \\
\hline CD at $5 \%$ & 3.143 & 19.768 & 0.928 & 2.224 & 0.303 & 1.158 & 6.962 & 4.434 & 0.076 & 0.997 \\
\hline
\end{tabular}

* Significant at $5 \%$ level; ** Significant at $1 \%$ level 
Table.4 Range of heterosis and number of crosses showing significant heterosis in desirable direction

\begin{tabular}{|c|c|c|c|c|c|c|c|}
\hline \multirow[t]{2}{*}{ S. No. } & \multirow[t]{2}{*}{ Character } & \multicolumn{2}{|c|}{ Mid parent heterosis } & \multicolumn{2}{|c|}{ Heterobeltiosis } & \multicolumn{2}{|c|}{ Standard heterosis } \\
\hline & & Range & No. of crosses & Range & No. of crosses & Range & No. of crosses \\
\hline 1. & $\begin{array}{l}\text { Days to } 50 \text { per cent } \\
\text { flowering }\end{array}$ & -19.00 to 20.85 & 25 & -28.99 to 20.42 & 38 & -12.75 to 16.78 & 4 \\
\hline 2. & Plant height (cm) & -11.12 to 58.39 & 28 & -25.26 to 53.92 & 4 & -11.92 to 33.61 & 5 \\
\hline 3. & $\begin{array}{l}\text { Number of productive } \\
\text { tillers per plant }\end{array}$ & -74.24 to 30.37 & 0 & -75.38 to -7.00 & 0 & -57.81 to 45.31 & 0 \\
\hline 4. & Panicle length $(\mathrm{cm})$ & -19.63 to 35.26 & 28 & -30.68 to 32.85 & 11 & -33.24 to 9.57 & 0 \\
\hline 5. & Panicle width $(\mathrm{cm})$ & -26.86 to 62.26 & 12 & 42.31 to 21.13 & 1 & -23.68 to 21.05 & 4 \\
\hline 6. & 1000-grain weight $(\mathrm{g})$ & -21.61 to 48.50 & 27 & -28.53 to 44.58 & 14 & 10.45 to 95.91 & 54 \\
\hline 7. & Fodder yield (t/ha) & -76.00 to 199.68 & 8 & -82.56 to 96.67 & 2 & -64.83 to 64.66 & 5 \\
\hline 8. & Total biomass (t/ha) & 21.46 to 417.60 & 42 & -56.84 to 474.18 & 22 & -70.24 to 286.11 & 5 \\
\hline 9. & Harvest index & -76.92 to -30.84 & 0 & -67.03 to 57.52 & 1 & -75.38 to -30.84 & 0 \\
\hline 10. & Grain yield (t/ha) & -71.11 to 304.37 & 18 & -71.11 to 128.00 & 6 & -71.11 to 34.44 & 0 \\
\hline
\end{tabular}

Table.5 Top ranking genotypes based on per se performance, gca, sca and heterosis

\begin{tabular}{|c|c|c|c|c|c|c|c|}
\hline \multirow[t]{2}{*}{ Character } & \multicolumn{2}{|c|}{ Best general combiners } & \multicolumn{2}{|l|}{ Best specific combiners } & \multirow{2}{*}{$\begin{array}{l}\text { Mid parent } \\
\text { heterosis }\end{array}$} & \multirow[t]{2}{*}{ Heterobeltiosis } & \multirow{2}{*}{$\begin{array}{l}\text { Standard } \\
\text { heterosis }\end{array}$} \\
\hline & $\begin{array}{l}\text { Based on } \\
\text { gca }\end{array}$ & $\begin{array}{l}\text { Based on per se } \\
\text { performance }\end{array}$ & Based on sca & $\begin{array}{l}\text { Based on per se } \\
\text { performance }\end{array}$ & & & \\
\hline $\begin{array}{l}\text { Days to } 50 \text { per } \\
\text { cent flowering }\end{array}$ & $\begin{array}{l}\text { ICMA04999 } \\
2381 \\
237\end{array}$ & $\begin{array}{l}\text { ICMA } 97111 \\
\text { ICMA } 04999 \\
2306,2349\end{array}$ & $\begin{array}{l}\text { ICMA } 97111 \times 2386 \\
\text { ICMA } 04999 \times 2310 \\
\text { ICMA } 04999 \times 2311\end{array}$ & $\begin{array}{l}\text { ICMA } 04999 \times 2310 \\
\text { ICMA } 04999 \times 2311 \\
\text { ICMA } 04999 \times 2318\end{array}$ & $\begin{array}{l}\text { ICMA } 04999 \times \\
2310 \\
\text { ICMA } 97111 \times \\
2386 \\
\text { ICMA } 04999 \times \\
2311\end{array}$ & $\begin{array}{l}\text { ICMA } 97111 \times \\
2328 \\
\text { ICMA } 04999 \\
\times 2328 \\
\text { ICMA } 04999 \times \\
2310\end{array}$ & $\begin{array}{l}\text { ICMA } 04999 \times \\
2310 \\
\text { ICMA } 04999 \times \\
2311 \\
\text { ICMA } 04999 \times \\
2318\end{array}$ \\
\hline Plant height (cm) & $\begin{array}{l}\text { ICMA } \\
97111, \\
2318 \text { (for } \\
\text { dwarfness) }\end{array}$ & $\begin{array}{l}\text { ICMA } 04999, \\
2329 \text { (for } \\
\text { dwarfness) } \\
2368,2325, \\
2382 \text { (for }\end{array}$ & $\begin{array}{c}\text { ICMA } 04999 \times 2311 \\
\text { ICMA } 97111 \times 2382 \\
\quad(\text { for dwarf types }) \\
\text { ICMA } 97111 \times 2311 \\
\text { ICMA } 04999 \times 2382\end{array}$ & $\begin{array}{l}\text { ICMA } 97111 \times 2349 \\
\text { ICMA } 97111 \times 2370 \\
\quad \text { (for dwarf types) } \\
\text { ICMA } 97111 \times 2311 \\
\text { ICMA } 04999 \times 2382\end{array}$ & $\begin{array}{l}\text { ICMA } 04999 \times \\
2329 \\
\text { ICMA } 97111 \times \\
2311 \\
\text { ICMA } 04999 \times\end{array}$ & $\begin{array}{l}\text { ICMA } 04999 \times \\
2329 \\
\text { ICMA } 97111 \times \\
2311 \\
\text { ICMA } 97111 \times\end{array}$ & $\begin{array}{l}\text { ICMA } 97111 \times \\
2311 \\
\text { ICMA } 04999 \times \\
2382 \\
\text { ICMA } 04999 \times\end{array}$ \\
\hline
\end{tabular}




\begin{tabular}{|c|c|c|c|c|c|c|c|}
\hline & & tallness) & (for tallness) & (for tallness) & 2330 & 2330 & 2329 \\
\hline $\begin{array}{l}\text { Total number of } \\
\text { productive tillers } \\
\text { per plant }\end{array}$ & $\begin{array}{l}2311 \\
2346 \\
2386\end{array}$ & $\begin{array}{l}2368 \\
2387 \\
2309\end{array}$ & 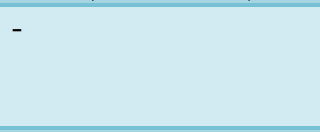 & $\begin{array}{l}\text { ICMA } 97111 \times 2311 \\
\text { ICMA } 97111 \times 2306 \\
\text { ICMA } 04999 \times 2318\end{array}$ & - & - & - \\
\hline $\begin{array}{l}\text { Panicle length } \\
\text { (cm) }\end{array}$ & $\begin{array}{l}\text { ICMA } \\
04999 \\
2368 \\
2364\end{array}$ & $\begin{array}{l}2325 \\
2327 \\
2370\end{array}$ & $\begin{array}{l}\text { ICMA } 04999 \times 2382 \\
\text { ICMA } 97111 \times 2311 \\
\text { ICMA } 04999 \times 2349\end{array}$ & $\begin{array}{l}\text { ICMA } 04999 \times 2382 \\
\text { ICMA } 04999 \times 2364 \\
\text { ICMA } 97111 \times 2368\end{array}$ & $\begin{array}{l}\text { ICMA } 97111 \times \\
2368 \\
\text { ICMA } 97111 \times \\
2330 \\
\text { ICMA } 97111 \times \\
2387\end{array}$ & $\begin{array}{l}\text { ICMA } 97111 \times \\
2368 \\
\text { ICMA } 97111 \times \\
2330 \\
\text { ICMA } 97111 \times \\
2387\end{array}$ & $\begin{array}{l}\text { ICMA } 97111 \times \\
2368 \\
\text { ICMA } 97111 \times \\
2330 \\
\text { ICMA } 97111 \times \\
2395\end{array}$ \\
\hline Panicle width (cm) & - & $\begin{array}{l}2365 \\
2370 \\
2328\end{array}$ & - & $\begin{array}{l}\text { ICMA } 97111 \times 2325 \\
\text { ICMA } 04999 \times 2394 \\
\text { ICMA } 97111 \times 2306\end{array}$ & $\begin{array}{l}\text { ICMA } 04999 \times \\
2337 \\
\text { ICMA } 04999 \times \\
2309 \\
\text { ICMA } 97111 \\
\times 2337\end{array}$ & $\begin{array}{l}\text { ICMA } 04999 \times \\
2337\end{array}$ & $\begin{array}{l}\text { ICMA } 97111 \times \\
2325 \\
\text { ICMA } 04999 \times \\
2394 \\
\text { ICMA } 97111 \times \\
2306\end{array}$ \\
\hline $\begin{array}{l}1000 \text { grain weight } \\
\text { (g) }\end{array}$ & $\begin{array}{l}\text { ICMA } \\
97111 \\
2352 \\
2306,2309\end{array}$ & $\begin{array}{l}\text { ICMA } 97111 \\
2349 \\
2342\end{array}$ & $\begin{array}{l}\text { ICMA } 97111 \times 2333 \\
\text { ICMA } 97111 \times 2394 \\
\text { ICMA } 04999 \times 2331\end{array}$ & $\begin{array}{l}\text { ICMA } 97111 \times 2333 \\
\text { ICMA } 97111 \times 2309 \\
\text { ICMA } 97111 \times 2394\end{array}$ & $\begin{array}{l}\text { ICMA } 04999 \times \\
2387 \\
\text { ICMA } 04999 \times \\
2337 \\
\text { ICMA } 04999 \times \\
2364\end{array}$ & $\begin{array}{l}\text { ICMA } 04999 \times \\
2364 \\
\text { ICMA } 04999 \times \\
2328 \\
\text { ICMA } 04999 \\
\times 2309\end{array}$ & $\begin{array}{l}\text { ICMA } 97111 \times \\
2333 \\
\text { ICMA } 97111 \times \\
2309 \\
\text { ICMA } 97111 \times \\
2394\end{array}$ \\
\hline Fodder yield (t/ha) & - & $\begin{array}{l}2368 \\
\text { ICMA } 97111 \\
2325\end{array}$ & $\begin{array}{l}\text { ICMA } 97111 \times 2310 \\
\text { ICMA } 04999 \times 2309 \\
\text { ICMA } 97111 \times 2311\end{array}$ & $\begin{array}{l}\text { ICMA } 97111 \times 2310 \\
\text { ICMA } 04999 \times 2309 \\
\text { ICMA } 04999 \times 2386\end{array}$ & $\begin{array}{l}\text { ICMA } 04999 \times \\
2309 \\
\text { ICMA } 04999 \times \\
2329 \\
\text { ICMA } 04999 \times \\
2348\end{array}$ & $\begin{array}{l}\text { ICMA } 04999 \times \\
2309 \\
\text { ICMA } 04999 \times \\
2348\end{array}$ & $\begin{array}{l}\text { ICMA } 97111 \times \\
2310 \\
\text { ICMA } 04999 \times \\
2309 \\
\text { ICMA } 04999 \times \\
2386\end{array}$ \\
\hline $\begin{array}{l}\text { Total biomass } \\
\text { (t/ha) }\end{array}$ & $\begin{array}{l}\text { ICMA } \\
04999\end{array}$ & $\begin{array}{l}2325 \\
2352 \\
2330\end{array}$ & ICMA $04999 \times 2348$ & $\begin{array}{l}\text { ICMA } 04999 \times 2325 \\
\text { ICMA } 04999 \times 2382 \\
\text { ICMA } 04999 \times 2386\end{array}$ & $\begin{array}{l}\text { ICMA } 04999 \times \\
2325 \\
\text { ICMA } 04999 \times \\
2348 \\
\text { ICMA } 04999 \times \\
2382\end{array}$ & $\begin{array}{l}\text { ICMA } 04999 \times \\
2309 \\
\text { ICMA } 97111 \times \\
2329 \\
\text { ICMA } 97111 \times \\
2310\end{array}$ & $\begin{array}{l}\text { ICMA } 04999 \times \\
2309 \\
\text { ICMA } 97111 \times \\
2329 \\
\text { ICMA } 97111 \times \\
2310\end{array}$ \\
\hline
\end{tabular}

Table. 5 (Contd.) 


\begin{tabular}{|c|c|c|c|c|c|c|c|}
\hline \multirow[t]{2}{*}{ Character } & \multicolumn{2}{|c|}{ Best general combiners } & \multicolumn{2}{|c|}{ Best specific combiners } & \multirow{2}{*}{$\begin{array}{l}\text { Mid parent } \\
\text { heterosis }\end{array}$} & \multirow[t]{2}{*}{ Heterobeltiosis } & \multirow{2}{*}{$\begin{array}{l}\text { Standard } \\
\text { heterosis }\end{array}$} \\
\hline & Based on gca & $\begin{array}{l}\text { Based on per } \\
\text { se } \\
\text { performance }\end{array}$ & $\begin{array}{l}\text { Based on } \\
\text { sca }\end{array}$ & $\begin{array}{l}\text { Based on per se } \\
\text { performance }\end{array}$ & & & \\
\hline $\begin{array}{l}\text { Harvest } \\
\text { index }\end{array}$ & $\begin{array}{l}\text { ICMA } 97111, \\
2365\end{array}$ & $\begin{array}{l}\text { ICMA } 97111 \\
2348 \\
\text { ICMA } 04999\end{array}$ & $\begin{array}{l}\text { ICMA } \\
97111 \times \\
2348\end{array}$ & $\begin{array}{l}\text { ICMA } 97111 \times \\
2348 \\
\text { ICMA } 97111 \times \\
2365 \\
\text { ICMA } 04999 \times \\
2333 \\
\text { ICMA } 97111 \times \\
2327 \\
\text { ICMA } 97111 \times \\
2332\end{array}$ & - & $\begin{array}{l}\text { ICMA } 04999 \times \\
2328\end{array}$ & - \\
\hline $\begin{array}{l}\text { Grain yield } \\
\text { (t/ha) }\end{array}$ & $\begin{array}{l}\text { ICMA } 04999 \\
2325,2396,2306, \\
2337,2348, \\
2394\end{array}$ & $\begin{array}{l}2348 \\
2332 \\
\text { 2331, 2370, } \\
\text { ICMA 97111 }\end{array}$ & $\begin{array}{l}\text { ICMA } \\
04999 \times \\
2309\end{array}$ & $\begin{array}{l}\text { ICMA } 04999 \times \\
2394 \\
\text { ICMA } 04999 \times \\
2348 \\
\text { ICMA } 97111 \times \\
2337 \\
\text { ICMA } 04999 \times \\
2309\end{array}$ & $\begin{array}{l}\text { ICMA } 04999 \\
\times 2309 \\
\text { ICMA } 04999 \\
\times 2328 \\
\text { ICMA } 04999 \\
\times 2333\end{array}$ & $\begin{array}{l}\text { ICMA } 04999 \times \\
2396 \\
\text { ICMA } 04999 \times \\
2309 \\
\text { ICMA } 04999 \times \\
2333\end{array}$ & - \\
\hline
\end{tabular}


Peng and Virmani (1990) reported possibility of interaction between positive alleles from good combiners and negative alleles from poor combiners in high $\times$ low or low $\times$ high combiner crosses and suggested for the exploitation of heterosis in $\mathrm{F}_{1}$ generation as their high yield potential would be unfixable in succeeding generations.

The estimates of heterosis revealed that, out of 60 crosses the cross ICMA $04999 \times 2309$ recorded significant heterosis over mid parent and better parent along with high sca effects in desirable direction for grain yield and fodder yield. The crosses ICMA $04999 \times$ 2310 and ICMA $04999 \times 2311$ recorded significant mid parent heterosis, heterobeltiosis and standard heterosis in addition to high sca effects for days to $50 \%$ flowering, indicating earliness in flowering. The crosses ICMA $04999 \times 2329$ and ICMA $97111 \times 2311$ recorded significant positive heterosis for plant height over mid parent, better parent and standard parent. These hybrids will help in production of tall genotypes to improve the fodder yield. The crosses ICMA $97111 \times 2368$ and ICMA $97111 \times 2330$ recorded significant heterosis over mid parent and better parent with lack of significant sca effect in desirable direction for panicle length. The crosses ICMA $04999 \times$ 2337 and ICMA $04999 \times 2364$ recorded high significant positive heterosis over mid parent and better parent for panicle width and 1000grain weight respectively. The crosses ICMA $04999 \times 2309$, ICMA $97111 \times 2329$ and ICMA $97111 \times 2310$ were best over better parent and standard check for total biomass. ICMA $97111 \times 2325$ showed high standard heterosis for panicle width. ICMA $04999 \times$ 2328 recorded high heterobeltiosis for harvest index. Similar results for yield and its components were earlier reported by Kanfany et al., (2018) for grain yield; Athoni et al., (2016) for panicle length, panicle width and fodder yield; Bhasker et al., (2017) for days to 50 per cent flowering and plant height; Acharya et al., (2017) for total biomass and harvest index. The range of heterosis and number of significant crosses are presented in Table 4.

In the present study, Table 5 revealed that there is a lack of relation between per se performance, sca effects and heterosis which means that the cross recording high sca effect may not have high heterosis. Hence consideration of these three criterion will be effective for selection of best cross combinations. Based on per se performance, sca effect and heterosis, the crosses ICMA $04999 \times 2309$ for grain yield and fodder yield; ICMA $97111 \times 2311$ for plant height; ICMA $97111 \times 2333$ for 1000 -grain weight; ICMA $04999 \times 2348$ for total biomass are the best cross combinations deduced from the study.

From the present investigation it can be concluded that all the characters are governed by non-additive gene action. The good combiner parents for different traits are 2325 for grain yield, panicle width, panicle length and 1000-grain weight; 2381 for days to 50 per cent flowering; 2349 for plant height; 2368 for panicle length and 1000-grain weight; 2365 for harvest index. These good combiner parents could be further used in the hybrid breeding programmes to produce better crosses or to develop better base/parent material.

The developed parental material could be used in breeding programmes for development of improved genotypes. The cross ICMA $04999 \times 2309$ recorded significant specific combing ability, mid parent heterosis and heterobeltiosis for grain yield and green fodder yield. Hence this cross was selected for dual purpose. In this study, a single cross did not record significant heterosis for majority of traits indicating the 
presence of more genetic variation in the parental material and possibility of genetic improvement through recurrent selection.

\section{References}

Acharya, Z.R., Khanapara, M.D., Chaudhari, V.B and Dobaria, J.D. 2017. Exploitation of heterosis in pearl millet [Pennisetum glaucum (L.) R. Br.] for yield and its component traits by using male sterile line. International Journal of Current Microbiology and Applied Sciences. 6 (12): 750-759.

Athoni, B. K., Boodi, I.H and Guggari, A.K. 2016. Combining ability and heterosis for grain yield and its componets in pearl millet [Pennisetum glaucum (L.) $\mathrm{R}$. Br.]. International Journal of Science and Nature. 7 (4): 786-794.

Bhadalia, A.S., Dhedhi, K.K., Joshi, H.J and Sorathiya, J.S. 2014. Combining ability studies through diallel analysis in pearl millet [Pennisetum glaucum (L.) R. Br.]. International Journal of Agricultural Science. 10 (1): 57-60.

Bharat, K.D and Dangaria, C.J. 2018. Diallel analysis for grain yield and component traits in pearl millet [Pennisetumglaucum (L.) R. Br.] under semi-arid condition of Gujarat. International Journal of Current Microbiology and Applied Sciences. 7 (7): 3942-3950.

Bhardwaj, R., Kaur, M., Sohu, R.S and Singh, D.P. 2015. Combining ability studies in pearl millet [Pennisetum glaucum (L.) R. Br.]. Forage Research. 41 (2): 78-84.

Bhasker, K., Shashibhushan, D., Krishna, K.M and Bhave, M.H.V. 2017. Studies on heterosis for grain yield and its contributing characters in hybrids of pearl millet [Pennisetum glaucum (L.) R. Br.]. International Journal of Plant and Soil Science. 18 (5): 1-6.
Gavali, R.K., Kute, N.S., Pawar, V.Y and Patil, H.T. 2018. Combining ability analysis and gene action studies in pearl millet [Pennisetum glaucum (L.) R. Br.]. Electronic Journal of Plant Breeding. 9 (3): 908-915.

Kanfany, G., Fofana, A., Tongoona, P., Danquah, A., Offei, S., Danquah, E and Cisse, N. 2018. Estimates of combining ability and heterosis for yield and its related traits in pearl millet inbred lines under downy mildew prevalent areas of Senegal. International Journal of Agronomy. 10: 1-2.

Kempthorne, O. 1957. An Introduction to Genetic Statistics. John Willey \& sons. Inc., New York. 458-471.

Khandagale, S.G., Sharma, V., Lone, R.P., Khandagale, V.G and Swamy, R.V. 2014. Combining ability analysis and gene action in pearl millet [Pennisetum glaucum (L.) R. Br.]. Electronic Journal of Plant Breeding. 5 (3): 445-450.

Krishnan, M. R. R., Patel. M.S and Gami, R.A. 2019. Combining ability and gene action analysis in pearl millet [ Pennisetum glaucum (L). R. Br.]. Chemical Science Review and Letters. 8 (30): 226-230.

Kumar, M., Gupta, P.C., Pawan, K and Heeralal, B. 2017. Assessment of combining ability and gene action for grain yield and its component traits in pearl millet [Pennisetumglaucum (L.) R. Br.]. Journal of Pharmacognosy and Phytochemistry. 6 (3): 431-434.

Ministry of Agriculture, 2017-2018. Government of India. http:// www.indiaagristat.com

Patel, B.C., Patel, M.P and Patel, J.A. 2018. Combining ability and gene action for grain yield and its attributing traits in pearl millet [Pennisetum glaucum (L.) R. Br.]. Electronic Journal of Plant Breeding. 9 (4): 1396-1402.

Patterson, H. D. and Williams, E. R. (1976): 
A new class of resolvable incomplete block designs. Biometrika, 63: 83-90.

Peng, Y.J and Virmani, S.S. 1990. Combining ability for yield and four related traits in relation to breeding in rice. Oryza. 27: 1-10.

Rao, N.G.P. (1972). Sorghum breeding in India-Recent developments of sorghum in seventies. Oxford and IBH Publishing Company, New Delhi. 101-142.

Saini, L.K., Solanki, K., Gupta, P.C., Saini, H and Singh, A.G. 2018. Combining ability studies for grain yield and component traits in pearl millet [Pennisetum glaucum (L.) R. Br.]. International Journal of Chemical Studies. 6 (1): 1939-1944.

Shinde, G.C and Mehetre, S.S. 2014. Genetic analysis for yield and quality traits in forage pearl millet [Pennisetum glaucum
(L.) R. Br.]. Forage Research. 41 (2): 78-84.

Singh, J and Sharma, R. 2014. Assessment of Combining Ability in Pearl Millet Using Line $\mathrm{x}$ Tester Analysis. Advances in Crop Science and Technology. 2 (147): 2

Solanki, K.L., Bhinda, M.S., Gupta, P.C., Saini, H and Saini, L.K. 2017. Combining ability and gene action studies for grain yield and component characters in pearl millet [Pennisetum glaucum (L.) R. Br.] under arid condition of Rajasthan. International Journal of Pure and Applied Science. 5 (4): 2121-2129.

Sprague, G.F and Tatum, L.A. 1942. General vs. specific combining ability in single crosses of corn. Agronomy Journal. 34: 923-932.

\section{How to cite this article:}

Bala Barathi, M., B. Vijaya Lakshmi, P. Sanjana Reddy and Nafeez Umar, Sk. 2020. Heterosis and Combining Ability Studies in Indigenous Collection of Pearl Millet Germplasm [Pennisetum glaucum (L.) R. Br.]. Int.J.Curr.Microbiol.App.Sci. 9(10): 2648-2660. doi: https://doi.org/10.20546/ijcmas.2020.910.319 